events that she might blame for her misfortune. The other control group was the Collaborative Perinatal Project population. Although these women had been interviewed during pregnancy and so had not had as much time as the other controls to forget, they too lacked the incentive the cases' mothers had to recall events in early pregnancy, and being a relatively underprivileged group (55\% Black or Puerto Rican ${ }^{1}$ ) they are less likely to have ever known what their body temperature was during any fever.

Secondly, Miller et al. suggest that published work favours the view that local outbreaks of febrile illness and of anencephaly tend to be associated-but two of the four papers they cite in this connection ${ }^{2,3}$ only mention associations involving about four anencephalics each, and the other $\mathrm{two}^{4,5}$ do not mention epidemics of febrile illness at all but deal exclusively with increases in the frequency of neural-tube defects which lasted several years. No mention is made of other published work in which several very large series of live and still births occurring 26-40 weeks after influenza epidemics (including well over a thousand anencephalics in all) were compared with births in the same places at other times in respect of the frequency of various malformations--with results that were completely negative for anencephaly. ${ }^{6,7}$

Another population that seems to be at increased risk of hyperthermia but not of anencephaly is that of Finland, the home of the sauna, where less than $0.6 / 1000$ births were reported to be anencephalic. ${ }^{8}$

Especially in the light of the research on other species cited by Miller et al., their hypothesis that hyperthermia can cause anencephaly in man may merit further testing; but the above features of their control groups and of the population studies cited make this hypothesis less convincing than Miller et al. suggest.

Department of Community Medicine,

University of Manchester,

Manchester M13 9PT

IAN LECK

\section{PLASMAPHERESIS IN RAYNAUD'S DISEASE}

SrR,-Dr Talpos and his colleagues (Feb. 25, p. 416) report dramatic improvement after plasmapheresis in four cases of Raynaud's phenomenon with ulceration of the digits. Since this syndrome is seen in a heterogeneous group of conditions of which sclerodactyly with telangiectasia and calcinosis (C.R.S.T. syndrome) ${ }^{9}$ is perhaps the most common, it would be helpful in evaluating the report to have more information about the clinical signs and the results of the tests for autoimmune antibodies in the patients.

Southampton General Hospital,

Southampton SO9 $4 \mathrm{XY}$

E. D. ACHESON

** This letter has been shown to Dr Talpos and his colleagues whose reply follows.-ED.l.

SIR,-Two of our five patients were diagnosed as having scleroderma on the basis of constitutional symptoms, severe Raynaud's phenomenon, abnormal osophageal motility and abnormal antinuclear-antibody patterns. Neither shows the C.R.S.T. variant of scleroderma to which Professor Acheson refers. We have yet to detect any underlying disease in the other three patients.

1. Heinonen, O. P., Slone, D., Shapiro, S. Birth Defects and Drugs in Pregnancy. New York, 1977.

2. Haynes, S. G., Gibson, J. B., Kurland, L. T. Neurology, Minneap. 1974, 24, 691.

3. Solowy, M. K., Shepard, F. M. Clin. Pediat. 1971, 10, 43

4. MacMahon, B., Yen, S. Lancet, 1971, i, 31

5. Leck, 1. ibid. 1966, ii, 791.

6. Leck, I. Br. F. prev. soc. Med.1963, 17, 70.

7. Leck, I. H.S.M.H.A.HLth Rep. 1971, 86, 265.

8. Klemetti, A. Saxén, L Hlth Serv Res natn Board Hlth Finland, 1970,9.

9. Winterbauer R. H. Bull. Fohns Hopktns Hosp. 1964, 114, 361.
The nomenclature of small-vessel disease of the extremities is confused. The terms Raynaud's disease and Raynaud's phenomenon are often used interchangeably, and some writers attempt to divide them into primary and secondary to classify better the clinical signs we so easily recognise.

All five patients have minor increase of at least one of the three immunoglobulins which we measure (IgA, IgG, and $\operatorname{IgM}$ ). These increases average about $15 \%$ above the upper limit of normal with a maximum increment of less than twice normal. Caution is necessary in interpreting this, however, because we have not determined what abnormalities, if any, would be present in a group of matched patients with cutaneous ulcers but without Raynaud's symptoms. Other routine tests have been unremarkable.

Clinically the five patients are slowly regressing to their pretreatment state, although they all are much improved. One patient with scleroderma who underwent plasmapheresis last summer for pregangrenous changes of an entire index finger eventually lost a small amount of finger pulp. She had a further three plasma exchanges, all averaging $70 \%$ total plasma volume, when her index finger began to deteriorate with the winter cold. Since then her condition has stabilised.

The clinical course of these patients leads us to believe that plasmapheresis is a valuable form of therapy for certain patients. Better treatment will be forthcoming when the mechanism for the efficacy of this therapy has been elucidated. We hope to answer some of these questions at the conclusion of our formal trial.

Departments of Biomedical Engineering and Hamatology, King's College Hospital Medical School,

London SE5 8RX

and Department of Surgery,

University of Michigan,

Ann Arbor, Michigan, U.S.A.

G. B. TALPoS

L. T. CotTON

J. M. WHITE

M. HORROCKS

\section{IMMUNE COMPLEXES AND PROGNOSIS OF HUMAN BREAST CANCER}

SIR,-We have found ${ }^{1}$ that sequential determination of circulating immune complexes (c.I.c.) confirms the clinicopathological prognosis in breast cancer. In patients with good prognosis (no lymph-node involvement detectable at mastectomy) C.I.C. values fell to normal range twelve months after the operation whilst the c.I.c. value remained high in patients with poor prognosis (metastases in apical axillary and/or internal mammary lymph-nodes at mastectomy) and in those who died from cancer within 22 months of the operation.

We now have results on 83 patients and have found a similar pattern of c.I.c. values varying according to clinicopathological prognosis or death (figure a).

When C.I.c. levels were evaluated by outcome (dead or alive) 12-48 months after mastectomy (figure b) the correlation with prognosis assessed clinicopathologically had disappeared. By contrast, patients both with good and poor prognosis who are still alive displayed preoperatively similar c.I.c. levels which decreased postoperatively to almost normal levels. Patients who died during the follow-up period, had, both preoperatively and postoperatively, C.I.c. levels $(P<0.05)$ significantly higher than those of the survivors. Again, there was no difference between patients with good and poor prognosis. Furthermore, the postoperative decrease in c.I.c. was not significant.

Figure $c$ covers patients followed up for 24 months after mastectomy and those of intermediate prognosis (metastases only in lower axillary lymph-nodes); patients given chemotherapy within 12 months of mastectomy have been excluded because this treatment reduces c.I.c. levels in animals. (unpublished). Regardless of clinicopathological staging, patients who

1. Höffken, K., Meredith, I. D., Robins, R. A., Baldwin, R. W., Davies, C. J., Blamey, R. W. Br. med. F., 1977, ii, 218.

2. Zubler, R. H., Lange, G., Lambert, P. H., Miescher, P. A. f. Immun. 1976, 116, 232 . 